\title{
Action répétitive et action répétée : aspect et pluralité verbale dans la réduplication en émérillon
}

Françoise Rose*

L'émérillon ${ }^{1}$, comme de nombreuses langues amérindiennes, connaît une morphologie relativement riche sur les verbes : des indices de personne, des affixes de voix, de négation, de TAM et des clitiques de TAM. Cette morphologie verbale ne se réduit pas à la simple concaténation de morphèmes possédant chacun leur forme et leur sens propres, mais inclut également le processus de réduplication par lequel une partie ou la totalité d'une base est recopiée puis affixée à cette même base avec une certaine fonction sémantique.

L'émérillon connaît de manière typique pour une langue tupi-guarani deux modèles de réduplication qui participent tous deux au sens de la répétition. Par ailleurs, ce type de catégories grammaticales est de nos jours souvent traité sous le thème de " pluralité verbale ». Partant du constat que le sémantisme de ce que l'on pourrait appeler grossièrement la « répétition » est rarement décrit avec précision, ni dans les études sur l'aspect, ni dans les études sur la pluralité verbale, et que l'émérillon possède deux outils pour exprimer des nuances distinctes de la répétition, dans cet article, nous entendons distinguer avec précision les sens voisins de ces deux modèles de réduplication, en reprenant la terminologie de Cusic (1981) de pluralité verbale interne/externe à l'événement (autrement dit d'action répétitive/action répétée), sur fond de distinctions sémantiques relatives aux domaines de l'aspect et de la pluralité verbale.

Dans cet article, nous délimiterons dans un premier temps le phénomène de réduplication en émérillon et nous nous focaliserons sur la réduplication des verbes. Dans un second temps, nous amènerons une discussion sur la répétition comme catégorie aspectuelle et comme catégorie de la pluralité verbale afin de clarifier la terminologie utilisée. Enfin, dans une troisième partie, nous décrirons avec détails les deux modèles de réduplication de l'émérillon: l'un spécialisé dans l'expression de la pluralité interne à l'événement, l'autre plutôt dans la

* CELIA (IRD/CNRS). Nous remercions pour leurs précieux commentaires les lecteurs anonymes, ainsi que Brenda Laca, Patricia Cabredo Hofherr, Antoine Guillaume, Alice Vittrant et Sylvie Voisin.

${ }^{1}$ L'émérillon est une langue tupi-guarani parlée en Guyane française par environ 400 locuteurs. Les exemples cités dans cet article sont essentiellement issus de textes enregistrés lors de séjours sur le terrain. 
pluralité externe à l'événement, avant de terminer sur des considérations d'ordre diachronique qui viennent remettre en cause cette distinction.

\section{DELIMITATION DU PHENOMENE DE REDUPLICATION EN EMERILLON}

\subsection{Le processus de réduplication}

La réduplication sera comprise ici comme la copie d'une partie ou de la totalité d'un mot en tant que processus morphologique.

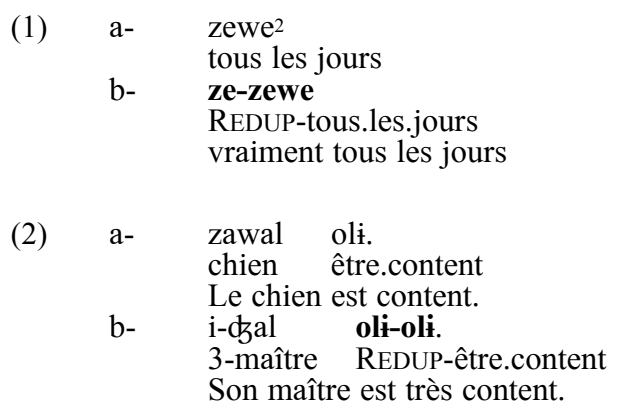

Le terme de réduplication ne concerne pas les racines formées d'une séquence de deux éléments identiques n'existant pas indépendamment. Moravcsik (1978) donne l'exemple du mot hongrois "papa" qui ne peut être analysé comme une réduplication de $p a$. Sont donc exclus du champ de la réduplication en émérillon des mots tels que:
(3) o-wiwi

$$
\text { 3-nettoyer }
$$$$
*_{0} \text {-wi }
$$$$
\text { Il nettoie. }
$$
(4) zaza *za
sœur aînée
(5) kosokosog *kosog
[idéophone pour le fait que quelqu'un ou quelque chose sorte de l'eau]

La réduplication comme processus morphologique diffère également $\mathrm{du}$ phénomène de répétition, que l'on observe dans les séquences de plusieurs réalisations d'un mot (le verbe omõga?u en (6)) séparées par une coupure

${ }^{2}$ Les transcriptions suivent les conventions de l'API. 
intonative. Ce critère de la constituance prosodique a été proposé parmi d'autres par Gil (2005) pour établir une distinction nette entre répétition et réduplication.

(6) o-mõ-ga?u, o-mõ-ga?u, o-mõ-ga?u.

3-CAUS-boire 3-CAUS-boire 3-CAUS-boire

Ils la font boire, ils la font boire, ils la font boire.

(7) o-itũ-itun, o-kusu-kusu(g)-katu-e?e, pug o-inu-inuy.

3-REDUP-sentir 3-REDUP-laver-bien-INTENS poser 3-REDUP-mettre

Il les sent, les lave bien et les repose chaque fois.

Le présent article se consacre donc à la réduplication envisagée comme un processus morphologique copiant une partie ou la totalité d'un morphème existant par ailleurs dans la langue et l'affixant à ce même morphème, comme illustré dans l'exemple précédant par les trois formes rédupliquées. En émérillon, la réduplication s'applique à divers types de bases morphosyntaxiques.

\subsection{Nature des bases morphosyntaxiques auxquelles la réduplication s'applique}

La réduplication en émérillon porte sur la plupart des parties du discours ${ }^{4}$ : les verbes (7), les adverbes (1), les noms quand ils prédiquent (nominoïdes (8) ou prédicats nominaux à sens possessif (9)), les prédicats à sens adjectival (10) et les numéraux (11). La forme rédupliquée appartient toujours à la même catégorie morphosyntaxique que la forme source.
(8) nipẽ-l-ehe oli-oli-o.
pain-RELN5-POSTP REDUP-être.content-CONT
Elle est très contente du pain.
(9) e-me-mebil
1SG-REDUP-enfant
J'ai beaucoup d'enfants.
(10) so-sə pila-ma?̃̃ o-bo-sale.
REDUP-être.gros poisson-REL 3-CAUS-être.salé
Ils salent les très gros poissons.
(11) moko-mokon z-ehe bijal o-anon. REDUP-deux REFL-à gibier 3-attacher
Il attache les pièces de gibier deux par deux.

\footnotetext{
${ }^{3}$ Ce verbe signifie plus précisément "boire de la bière de manioc en grande quantité". ${ }^{4}$ Pour la nature précise et argumentée des diverses parties du discours en émérillon, Cf. Rose 2003.

${ }^{5}$ Le "relationnel" est un morphème qui relie un verbe et son objet, une postposition et son objet ou un nom et son possesseur. Il n'est utilisé qu'avec certains verbes, postpositions ou noms déterminés dans le lexique.
} 
Le phénomène de réduplication est productif surtout sur les prédicats, donc statistiquement surtout sur les verbes. Dorénavant, cet article se focalisera donc sur la réduplication des prédicats, et plus précisément sur celle des verbes.

\subsection{Matrice phonologique de la réduplication en émérillon}

Il existe deux schémas de réduplication des verbes en émérillon, qui sont relativement complexes et peuvent tous deux s'appliquer aux mêmes bases. Dans le premier, la copie préfixée reprend la syllabe initiale de la base. On parlera de réduplication monosyllabique (12). Dans le deuxième, la copie reprend deux syllabes, le plus souvent les deux syllabes finales de la base : il s'agit de réduplication dissyllabique (13). La copie ne subit aucune modification particulière (ni changement vocalique, ni affixe supplémentaire...).

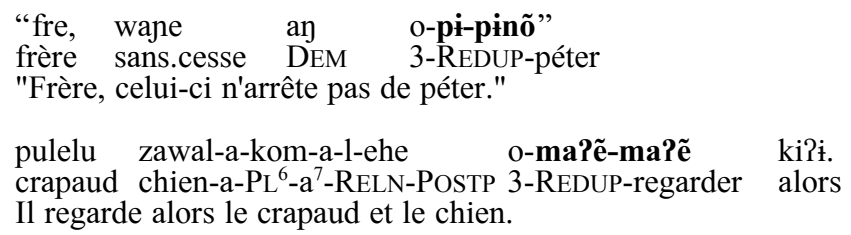

Les formes canoniques des racines émérillon sont constituées d'une ou deux syllabes (rarement plus), chaque voyelle constituant un noyau syllabique. Les syllabes fermées sont restreintes à la position finale dans le morphème ${ }^{8}$. Cette éventuelle consonne finale de la racine verbale est systématiquement exclue de la copie dans les deux schémas de réduplication. La réduplication ne copie ainsi que des syllabes ouvertes, comme dans l'exemple de réduplication dissyllabique suivant.

$$
\begin{aligned}
& \text { a-inu-inuy } \\
& \text { 1SG-REDUP-mettre musique } \\
& \text { Je mets toujours de la musique. }
\end{aligned}
$$

Des arguments pour analyser le mot résultant de la réduplication comme formé de la base précédée de la copie ainsi qu'une discussion sur le fait que l'émérillon puisse redoubler la syllabe initiale de la base alors que les autres

\footnotetext{
${ }^{6}$ Le suffixe de pluriel -kom marque ici la coordination entre les deux noms précédents. 7 Pour les besoins de l'article, l'analyse de ce morphème $-a$ de très large distribution n'est pas utile. Une analyse détaillée est proposée dans Rose 2003, chapitre 4.

${ }^{8}$ Les syllabes fermées n'apparaissent qu'en fin de mot, les règles morphophonologiques émérillon se chargeant de simplifier d'éventuelles séquences de consonnes à l'intérieur du mot, à la frontière entre morphèmes.
} 
langues tupi-guarani semblent toujours copier les syllabes finales sont proposées dans Rose (2005). ${ }^{9}$

De nombreuses racines verbales étant monosyllabiques, lors d'une réduplication dissyllabique, il est nécessaire de copier la syllabe précédant la racine, cette syllabe appartenant à un préfixe de voix ou de personne (o- en (15)), afin que la copie soit formée de deux syllabes $(\mathrm{C}) \mathrm{V}(\mathrm{C}) \mathrm{V}$.

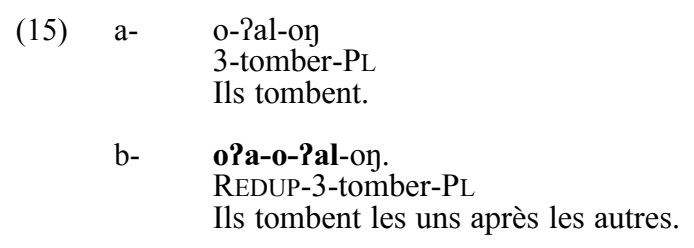

Après avoir défini la réduplication en émérillon (sa nature, les bases auxquelles elle s'applique, ses matrices phonologiques) et avant de discuter les sens de ces deux différents modèles de réduplication, nous tenons à présenter la littérature en notre connaissance sur la répétition comme appartenant à la fois aux catégories de l'aspect et de la pluralité verbale.

\section{LA REPETITION COMME CATEGORIE ASPECTUELLE ET COMME CATEGORIE DE LA PLURALITE VERBALE}

Le processus de réduplication qui s'applique aux prédicats de l'émérillon a une fonction purement grammaticale et ne sert pas à créer de nouvelles unités lexicales. Il s'agit d'un des moyens d'expression clairement iconique de l'itération (16) et de la distribution, comme en (17) où chaque agent réalise l'action indépendamment.

(16) nan e?i-e?i

ainsi REDUP-3.dire

Elle a répété ça (litt : elle a dit ainsi plusieurs fois).

(17) a?e-kom wila?a-uhu-1-ehe o-po-pol-on.

DEM-PL comou-gros-RELN-POSTP 3-REDUP-sauter-PL

Ceux-ci ont sauté du grand comou (arbre sp.).

Les études tupi-guarani présentent souvent une dichotomie très nette entre les sens des modèles de réduplication monosyllabique et dissyllabique (Jensen 1990

\footnotetext{
${ }^{9}$ Notre analyse de la forme rédupliquée comme une base précédée de sa copie se fonde essentiellement sur le principe d'économie. Il nous semble plus simple de poser que la base est rédupliquée en une copie qui la précède et qui répond à un modèle où chaque syllabe est monomoraïque (excluant donc toute consonne finale) plutôt que d'envisager que la base est intégralement rédupliquée en une copie qui la suit, et que c'est la base ellemême qui perd par la suite sa consonne finale sans qu'il s'agisse d'une nécessité morphophonologique de la langue.
} 
et 1998 sur la famille tupi-guarani, Seki 2000 sur le kamaiura, Rodrigues 1953 sur le tupinamba et Jensen 1989 sur le wayampi). La réduplication monosyllabique est dite exprimer une réalisation multiple du procès (des actions successives ou simultanées réalisées par une pluralité de sujets ou sur une pluralité d'objets) et la réduplication dissyllabique véhiculer un sens fréquentatif ou itératif.

Etant donné le caractère peu précis de la terminologie utilisée dans le domaine de la répétition, nous allons nous efforcer, dans cette section, de clarifier et préciser les diverses nuances sémantiques de la répétition. La répétition relève de deux paradigmes de catégorie grammaticale qui s'entrecroisent : l'aspect et la pluralité verbale. ${ }^{10}$

\subsection{La répétition comme catégorie aspectuelle}

L'aspect se définit comme la façon de voir la constitution temporelle interne d'une situation (Comrie 1976 : 2-3). La littérature sur l'aspect est plus prolixe sur des oppositions telles que perfectif/imperfectif que sur les différents sémantismes de l'itératif. Celui-ci est généralement défini comme la répétition d'une situation, "the successive occurrence of several instances of the given situation » (Comrie 1976). Il s'oppose au sémelfactif, qui dénote le fait qu'une situation n'ait lieu qu'une seule et même fois. Il est primordial de noter que si une situation est répétée un nombre de fois limité, alors toutes ces réalisations de la situation peuvent être vues comme une seule situation. Le problème central est donc celui de l'identification des événements, et par suite de leur éventuelle pluralité, « événement » étant défini par Comrie comme une situation dynamique vu comme un seul tout complet.

L'aspect itératif a cependant parfois retenu l'attention d'auteurs qui ont décomposé plus avant la notion d'itération. Chung et Timberlake (1985: 220222) ont établi quatre paramètres de variation de l'itération :

- la quantité des répétitions,

- la définitude du nombre de répétitions (un nombre de fois précis vs « plusieurs » fois)

- la régularité des répétitions

- le caractère individué et distinct des sous-événements (qui ont lieu à différentes occasions) par opposition au caractère collectif de sous-événements dont les diverses occurrences seraient groupées ensemble.

C'est cette dernière variable qui nous intéresse. Elle correspond grosso modo à l'aspect II de la typologie de Mel'cuk (2000, Vol II : 77-98). Cet auteur

10 Il est regrettable que l'étude de ces catégories n'ait pour l'instant pas fourni de terminologie claire qui soit partagée de manière consensuelle. Des termes comme par exemple itératif ou distributif ne recouvrent pas les mêmes réalités selon les auteurs. 
distingue 4 types d'aspects ${ }^{11}$ relevant de la quantification verbale. L'aspect II spécifie si le fait en question est concentré en un endroit et à un moment particulier ou s'il est distribué dans l'espace ou le temps. L'auteur discrimine ainsi 4 grammèmes d'aspect II, qui ne sont pertinents que pour des événements multiples comme plusieurs coups de feu :

- le concentratif, quand le fait en cause est présenté comme un tout, sans structure interne (salve)

- le distributif, quand le fait a lieu simultanément dans plusieurs endroits (fusillade)

- l'itératif, quand le fait a lieu à plusieurs moments successifs (rafale, tir continu)

- le distributif-itératif, quand le fait a lieu dans plusieurs endroits et à plusieurs moments (échanges de coups de feu).

Voyons maintenant comment la répétition peut être décrite comme participant à la pluralité verbale.

\subsection{La répétition comme catégorie de la pluralité verbale}

Le sémantisme de la réduplication en émérillon relève aussi du champ de la pluralité verbale, qui est conçue comme un domaine grammatical exprimant la pluralité des actions, à ne pas confondre avec l'accord sur le verbe du pluriel de ses arguments, qui est une catégorie nominale. Les marques entrant en jeu ont été désignées sous le terme de pluractional markers par Newman (1980). Les marqueurs de pluralité verbale renvoient à la multiplicité des actions, c'est-àdire à celle des participants, du temps ou de la localisation spatiale. Le domaine de la pluralité verbale intéressé par la multiplicité des actions dans le temps peut être rapproché du champ de la répétition dans le domaine aspectuel. La différence, selon notre intuition, est que la répétition comme marqueur pluricationnel va référer à des événements multiples (et donc discrets), alors que la répétition comme marqueur aspectuel va spécifier la constitution interne d'un seul et même événement ${ }^{12}$.

Corbett a consacré au nombre verbal un chapitre de son ouvrage typologique sur le nombre. Après avoir clairement séparé l'accord du pluriel nominal sur le verbe du véritable nombre verbal, il distingue deux types sémantiques de nombre verbal. Ceux-ci spécifient :

${ }^{11}$ L'aspect I est une catégorie dont les éléments spécifient le nombre de faits en question (neutre, multiplicatif, sémelfactif). L'aspect III est une catégorie dont les éléments spécifient l'étendue temporelle du fait en question (ponctuel, duratif, habituel). L'aspect IV est une catégorie dont les éléments spécifient si le fait en question est en train de se dérouler à un moment précis ou non (progressif, non-progressif).

12 Cette dernière idée n'est qu'une proposition de notre part, et n'est pas réellement explicitée dans la littérature existante. Cet article n'a pas l'ambition de redéfinir les catégories grammaticales d'aspect et de pluralité verbale, mais peut constituer une piste de réflexion dans cette direction. 
- le nombre des événements (exemples hausa, in Corbett 2000 : 246)

(18) naa aikee su

I send them

Je les ai envoyés (en même temps, au même endroit).

(19) naa a"'aikee su

I send.PL them

Je les ai envoyés à différents endroits au même moment / à différents moments au même endroit / à différents endroits à différents moments.

- le nombre des participants, qui opère sur une base absolutive (marquant sur les verbes unictanciels le nombre de l'unique argument et sur les verbes biactanciels celui du patient). (exemples huichol, Corbett 2000 : 247)

(20) nee waakana ne-mec-umi?ii-ri $\quad$ eeki J'ai tué le poulet pour toi.

(21) nee waakana-ari ne-mec-uqi?ii-ri eeki

1.SG poulet-PL 1.SG.S-2SG.O-tuer.PL-BenEF 2.SG J'ai tué les poulets pour toi.

Concrètement, il est possible qu'à l'intérieur d'une même langue, le même procédé grammatical puisse exprimer ces deux sémantismes, le sens d'un énoncé particulier étant alors une question d'interprétation. Notons aussi que de l'expression de la multiplicité des occurrences d'une action on infèrera souvent la multiplicité des participants.

La littérature sur la pluralité verbale formule directement la question de l'identification des événements. Ainsi, si Laca (sous presse, notre traduction) avoue que «les conditions d'identité des événements sont infiniment plus complexes que celles des objets », elle avance pour autant les trois paramètres principaux qui déterminent les conditions d'identité d'un événement: "les participants à l'événement, sa localisation spatiale et sa localisation temporelle ».

Par ailleurs, du fait que la pluralisation du verbe a souvent comme résultat un prédicat non borné et atélique, les études au croisement entre pluralité verbale et aspect nous paraissent prometteuses. C'est pourquoi nous nous baserons sur la terminologie proposée par Cusic (1981) dans un travail intitulé Verbal plurality and aspect.

2.3. La terminologie de Cusic au croisement entre aspect et pluralité verbale: répétition interne à l'événement vs. répétition externe à l'événement

Cusic (cité dans Wood 2002 entre autres) souligne un contraste important entre deux types sémantiques de répétition. D'un côté, la répétition interne à l'événement joue au niveau des phases de l'événement: elle implique un seul événement en une seule occasion, événement constitué de phases internes 
répétées. De l'autre côté, la répétition externe à l'événement joue au niveau de l'événement et répète des événements complets en une ou plusieurs occasions.

Cette distinction est résumée et illustrée par des exemples dans le tableau cidessous.

\begin{tabular}{|l|l|l|l|l|}
\cline { 2 - 5 } \multicolumn{1}{c|}{} & $\begin{array}{l}\text { niveau de } \\
\text { répétition }\end{array}$ & $\begin{array}{l}\text { nombre } \\
\text { d'événements }\end{array}$ & temps & $\begin{array}{l}\text { exemple } \\
\text { anglais }\end{array}$ \\
\hline singulier & & & bite \\
\hline $\begin{array}{l}\text { pluralité } \\
\text { interne } \\
\text { l'événement à }\end{array}$ & phase & un seul & $\begin{array}{l}\text { une seule } \\
\text { occasion }\end{array}$ & nibble \\
\hline $\begin{array}{l}\text { pluralité } \\
\text { externe à } \\
\text { l'événement }\end{array}$ & événement & plusieurs & $\begin{array}{l}\text { une ou } \\
\text { plusieurs } \\
\text { occasions }\end{array}$ & $\begin{array}{l}\text { bite over and } \\
\text { over/repeatedly }\end{array}$ \\
\hline \multicolumn{4}{|c|}{ Tableau 1: Résumé de la terminologie de Cusic }
\end{tabular}

Ce qu'ajoute Wood, c'est que les pluriactionnels peuvent aussi dénoter une distribution spatiale des événements, ou la pluralité des participants. Elle considère que les pluriels internes à l'événement requièrent l'identité des arguments d'une répétition à l'autre (les arguments pluriels doivent ainsi être collectifs), alors que les pluriels externes à l'événement peuvent avoir une interprétation distributive.

Nous utiliserons cette terminologie comme point de départ de la description qui suit des deux modèles de réduplication en émérillon (3.1 et 3.2). Nous nous attacherons aussi à observer l'effet de la réduplication sur l'aspect lexical du verbe concerné (3.3). Nous terminerons en une dernière étape par l'exposition de l'évolution diachronique vers une neutralisation de l'opposition entre ces deux sémantismes de répétition (3.4).

\section{DistinCTION SEMANTIQUE ENTRE REDUPLICATION MONOSYLLABIQUE ET} DISYLLABIQUE DES VERBES EMERILLON

Les deux modèles de réduplication de l'émérillon participent de l'expression de l'aspect $^{13}$ et de la pluralité verbale, plus précisément de la pluralité des

13 L'aspect est exprimé par diverses catégories morphologiques en émérillon: par des affixes TAM de prédicats (1), mais aussi par des clitiques, qui sont soit des affixes du syntagme prédicatif liés généralement au dernier élément du syntagme prédicatif (2), soit des "particules", clitiques liés à n'importe quel type de constituant en émérillon (3).

(1) o-poan-ba mididgu-nam, kija o-ba?e.

3-filer-COMPLF coton-quand hamac 3-faire

Quand elle a fini de filer tout le coton, elle fait le hamac.

(2) o-ho i-koti-n.

3-aller 3-chez-CONT

Il va chez lui.

(3) o-pol-e?e $\quad$ it-b o-?a-n. 
événements sur l'axe temporel. «Dans la pluractionalité temporelle, la multiplicité des événements est associée à une multiplicité d'intervalles temporels dans laquelle les événements se produisent, c'est-à-dire à une structure temporelle particulière qui indique comment une pluralité d'événement se distribue dans le temps.» (Laca, sous presse, notre traduction). Cette définition souligne à quel point la pluractionalité temporelle se rapproche de l'aspect. Réduplication monosyllabique et réduplication dissyllabique en émérillon exprimant deux types différents de répétition, nous allons tenter d'éclaircir, à partir de leur étude comparative, l'appartenance de la position de la répétition à l'aspect ou à la pluralité verbale. La distinction formelle entre les deux processus de réduplication de l'émérillon semble recouper clairement la distinction sémantique proposée par Cusic entre répétition interne à l'événement et répétition externe à l'événement.

\subsection{La réduplication monosyllabique : une répétition interne à l'événement}

La réduplication monosyllabique de l'émérillon nous paraît correspondre de manière claire au sémantisme de répétition interne à l'événement. Elle pourrait aussi suggérer la distributivité, c'est-à-dire le fait que l'action décrite est également vraie pour différents participants, lieux ou temps. Cependant, même si l'on peut envisager de décrire certains exemples en termes de distributivité des sujets $(22)(23)$ ou des objets (24)(25), et de simultanéité ou successivité des actions, de nombreux exemples aux participants singulier tels (26) à (31) montrent que ce n'est pas la notion de distribution qui est en jeu, mais celle d'événement unique, comportant des phases répétitives.
(22) amõ kito-kom õ-hẽ-hem.
autre grenouille-PL 3-REDUP-sortir
Les autres grenouilles sortent.
(23) tapug ze-kapilel-ne?i-b o-po-pol o-ho-y. plouf REFL-derrière-CONTRAST eau-dans 3-REDUP-sauter 3-aller-PL Elles plongent dans l'eau l'une derrière l'autre. $\begin{array}{ll}\text { (24) "e-ku-kusu(g)-katu } & \text { ay ba?e-kom" } \\ \text { 2SG.IMPER-REDUP-laver-bien } & \text { DEM chose-PL }\end{array}$ "Lave bien ces choses (des assiettes)".
(25) wasei-tukũ?ã-ne o-no-nopẽ o-ba?e ta?ay-am. wasai-pousse-CONTRAST 3-REDUP-tresser 3-faire mannequin-TRANSL Il tressa et tressa des palmes de wasai pour faire un mannequin.

\footnotetext{
3-sauter-INTENS eau-dans 3-tomber-PL
}

Elles replongent encore dans l'eau. 
(26) o-daio o-ho, o-ze-wa-pikikilig14 o-ho. 3-pleurer 3-aller 3-REFL-visage-REDUP.frotter 3-aller Il part en pleurant, il part en essuyant les larmes sur son visage (litt : en frottant-frottant son visage).

(27) "fre, wane an o-pi-pinõ"

frère sans.cesse DEM 3-REDUP-péter

"Frère, cette bête ne fait que péter" (dit le frère maladroit, alors qu'ils chevauchent un aigle géant en vol)

(28) "e-siluwa-bo-botug, fre!"

2SG.IMP-fesses-REDUP-cogner frère

"Tapote ses fesses, frère"

(29) nana-ne o-bo-?i-?i.

ananas-CONTRAST 3-CAUS-REDUP-être.petit

Elle découpait de l'ananas en petits morceaux.

(30) o-ze-zeka tupa-wəl.

3-REDUP-se.casser lieu-vide

La boîte s'est cassée en mille morceaux.

(31) ãduça wila o-su-su?u

rat bois 3-REDUP-mordre

Le rat a rongé le bois.

Ce qui relie tous ces exemples est que l'action répétée tient en un seul intervalle temps (on n'envisage pas d'intervalles vides entre les répétitions) : la réduplication monosyllabique introduit ainsi une lecture durative. De plus, l'action est réalisée en un seul lieu, ce qui constitue les principaux réquisits pour envisager ainsi un événement unique et homogène. Ce type de répétition doit ainsi être envisagé comme une catégorie aspectuelle. Cette analyse correspond à la position « caractère collectif de sous-événements dont les diverses occurrences seraient groupées ensemble » sur la quatrième variable proposée par Chung et Timberlake, et au concentratif de Mel'cuk. On pourrait représenter schématiquement le sémantisme de telles phrases ainsi :

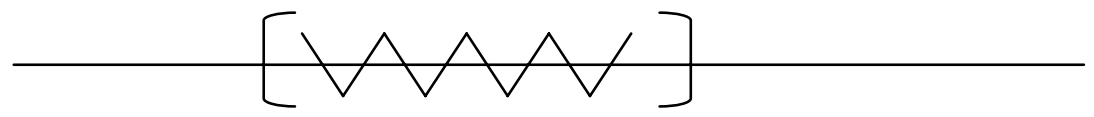

Schéma 1 : Représentation de la répétition interne à l'événement

14 Nous ne pouvons pas expliquer que la syllabe initiale $p \dot{i}$ ne soit pas la syllabe rédupliquée. Sur le plan sémantique qui nous préoccupe ici, cette forme est néanmoins représentative de la réduplication monosyllabique. 
L'unicité de l'événement résultant est clair dans l'exemple suivant, où le verbe rédupliqué se voit accoler une particule $-(e)$ ?e intensive qui exprime précisément ici une nouvelle occurrence de l'événement, "de nouveau ». Cette particule affirme l'existence d'un unique événement, tout en présupposant qu'une situation similaire a eu lieu dans un intervalle temporel antérieur ${ }^{15}$. C'est donc l'ensemble de l'événement, lui-même constitué de phases répétées, qui se reproduit, comme illustré par le schéma 2.
nana idgu-pe.
3-CAUS-REDUP-être.petit-INTENS ananas 3-pour Elle leur coupe de nouveau de l'ananas.

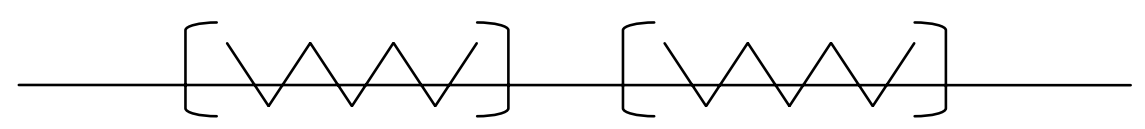

\section{Schéma 2 : Représentation d'une itération d'un événement à répétition interne}

L'interprétation distributive qu'il était possible d'avoir pour les exemples (22) à (25) n'est donc pas dominante. Au contraire, il semble que c'est l'unicité de l'événement qui est primordiale. Elle tend plutôt à donner une lecture " collective » des participants à l'action, qui ne sont pas différenciés les uns des autres (sauf en (23) à cause du groupe postpositionnel de succession spatiale). Cette remarque est conforme avec la corrélation proposée par Wood plus haut, selon laquelle le pluriel interne à l'événement nécessite l'identité des arguments d'une répétition à l'autre (Cf. 2.3).

On remarquera par ailleurs que de nombreux exemples impliquent une destruction progressive de l'objet. C'est ce que García-Medall (2003, p. 33) appelle «désintégration de l'objet ${ }^{16}$ dans sa typologie de la réduplication dans les langues amérindiennes.

Enfin, notons que la distribution de l'action dans l'espace utilise le mécanisme de la réduplication monosyllabique, mais pas sur le verbe dénotant l'action. C'est le verbe «tourner» qui est affecté, en tant que verbe final à sens directionnel d'une série verbale ${ }^{17}$.
(33) a-kaiu a-wa-wag. 1SG-boire(de la bière en quantité) 1SG-REDUP-tourner Je bois(de la bière de manioc) à droite à gauche

(34) o-ekal o-wa-wag-on.

15 Cf. l'ex (3), note 13, p.9.

16 "desintegración objetual"

17 Dans d'autres positions que celle de verbe final d'une série, le verbe wag rédupliqué en wawag à le sens de « se.promener». 
3-chercher 3-REDUP-tourner-PL

Ils le cherchent de tous côtés.

\subsection{La réduplication dissyllabique : une répétition externe à l'événement}

La réduplication dissyllabique rend l'idée d'une répétition du processus en plusieurs occurrences discrètes, séparées par des intervalles où le processus n'est pas actif. Afin de souligner la différence entre les deux types de réduplication de l'émérillon, reprenons deux verbes qui ont servi à illustrer la réduplication monosyllabique : hem « sortir », vu en (22) et kusug « laver » en (24).

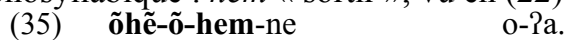

REDUP-3-sortir-CONTRAST 3-tomber

Il ressort encore et tombe.

(36) o-itũ-itun, o-kusu-kusu(g)-katu-e?e, pug o-inu-inuy. 3-REDUP-sentir 3-REDUP-laver-bien-INTENS poser 3-REDUP-mettre Il les renifle, les lave bien et les repose chaque fois.

Notons bien que dans ces deux exemples, il n'est pas question de distribution des participants. Dans l'exemple (35), l'agent est un jeune homme qui tente de tuer un ogre en lui labourant le ventre à coups de couteau (de l'intérieur) chaque fois qu'il se fait avaler par lui. Naturellement, il sort régulièrement du ventre de l'ogre par le conduit intestinal. La même action de «sortir » se reproduit donc avec le même participant, de manière successive mais séparée par des intervalles où l'action n'a pas lieu: les intervalles où le héros se fait avaler, est dans le ventre, donne des coups de couteau... De même, l'exemple (36) comparé à l'exemple (24) incarne parfaitement la distinction entre les deux types de réduplication : ces deux exemples sont issus du même texte et concernent le même agent (un homme) et le même patient (les assiettes). L'exemple (24) décrit les faits du héros lors de sa première soirée chez un monstre : il lave la vaisselle, constituée d'un certain nombre d'assiettes, et cette action répétitive est exprimée par la réduplication monosyllabique de (24). Le personnage va rester quelque temps chez le monstre, et va reproduire cette action régulièrement. Mais ce n'est pas la seule action du personnage : l'action de laver la vaisselle est intermittente, entrecoupée de séances où il renifle la vaisselle, la range, et d'autres où il mange. On a donc en (36) une répétition de l'action sur divers intervalles de temps séparés : c'est une répétition externe à l'événement.

Voici quelques autres exemples.

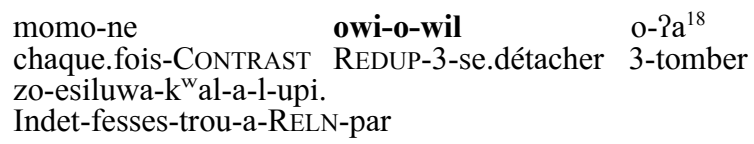

${ }^{18}$ L'action de tomber est très souvent exprimée par cette série verbale en émérillon : le verbe wil exprime le détachement initial et le verbe ?al la chute proprement dite. 
Il retombe chaque fois par le trou des fesses.

(38) o-ho-iwəl wilakala keił. mokone oke-o-kel 3-aller-CL Dieu alors quelques REDUP-3-dormir Dieu part alors quelques jours.(Litt: Dieu part. Il dort plusieurs fois pendant quelque temps. ${ }^{19}$ )

(39) awa-tipo e-l-emi?õ o-ba?e-ba?e-ma?ẽ? ${ }^{20}$ qui-INTER.EXCL 1SG-RELN-repas 3-REDUP-faire-Rel Qui donc me fait toujours à manger ? (à chaque fois que je pars chasser).

(40) "nan-a-iwəl-a-sipo o-tui-tui", ainsi-a-CL-a-EXCL 3-REDUP-être "C'est donc ainsi qu'elle fait régulièrement !", dit-il.

(41) ou si?e-si-?e-katu-nam, ...

oui REDUP-1INCL-dire-bien-si

Si nous disons "oui" à chaque fois, ...

Cette description d'une répétition externe à l'événement rejoint l'itératif de Mel'cuk (Cf. 2.1.1) et le fréquentatif de Van Geenhoven (2004: 154) décrit en termes de procès réalisés par intermittence, sur une succession d'intervalles temporels ne se chevauchant pas. On pourrait schématiser ce type de répétition comme suit (les tracés horizontaux symbolisant des états ou activités (atéliques), les barres obliques des actions uniques et les V des actions cycliques, Cf. Talmy, 1985).

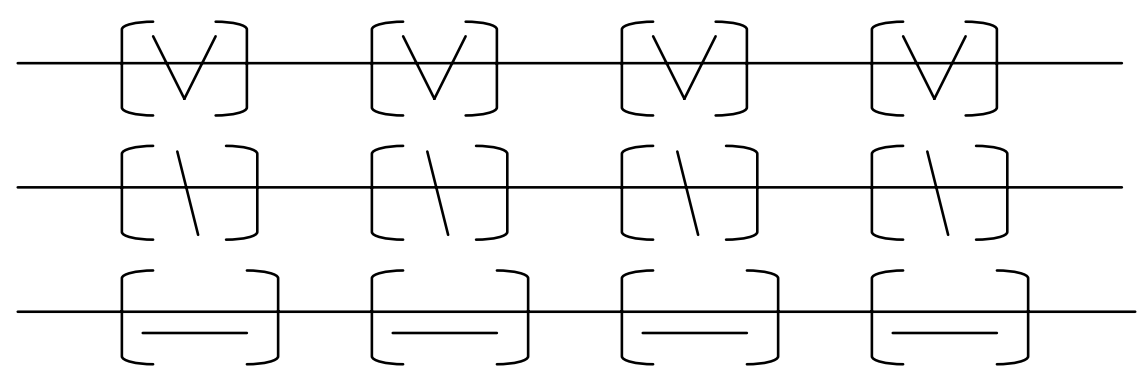

Schéma 3 : Représentation de la répétition externe à l'événement

${ }^{19}$ Cette périphrase est systématiquement utilisée pour préciser la durée d'un séjour.

${ }^{20}$ Les deux exemples suivants apparaissent dans un texte où un homme vit seul avec un singe. A chaque fois qu'il revient de la chasse, nourriture et boisson l'attendent à la maison. Il se demande qui prépare ces mets à chaque fois. Un jour, il fait sembler de partir à la chasse pour épier (en réalité, c'est une femme déguisée avec une dépouille de singe qui lui prépare ses collations). 
Alors que dans la réduplication monosyllabique, le changement de la quantité de l'événement est de l'ordre de l'extension (lecture durative), dans la réduplication dissyllabique, il est de l'ordre de la réplique (lecture fréquentative)- ce qui nous fait considérer ce dernier type de réduplication comme une expression de la pluralité verbale. Il est important de noter que dans la répétition externe à l'événement, chaque occurrence du procès répété est conçue comme un événement propre. Cette affirmation est soutenue par l'usage des idéophones dans l'exemple suivant, où chaque idéophone tapug illustre une action de plonger.

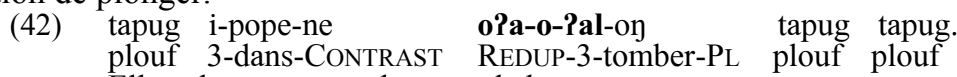
Elles plongent et replongent dedans.

La multiplication d'un procès identique peut mener à un sémantisme habituel, comme précédemment en (39) et (40), et dans l'exemple ci-dessous, énoncé résultant d'une tricherie dans un débat entre le jaguar et le tamanoir sur leurs habitudes alimentaires. Le jaguar échange ses excréments avec ceux du tamanoir afin que ce dernier se croie carnivore et tue ainsi du gibier dont le jaguar saura profiter.

$\begin{array}{ll}\text { (43) } & \text { a?u-a-Pu-sikəl } \\ & \text { REDUP-1-manger-CL } \\ & \text { Je mange donc de la viande ! }\end{array}$

Selon Wood, la répétition externe à l'événement peut avoir une interprétation distributive (sur les participants). En terme de distribution, c'est clairement la distribution dans le temps qui est primordiale dans la pluralité verbale externe de l'émérillon: le processus est distribué dans le temps avec généralement les mêmes participants à chaque occurrence du processus. Les exemples où l'action peut être distribuée sur plusieurs patients tout en étant distribuée dans le temps (ce qui implique que l'action affecte les patients l'un après l'autre) semblent en fait peu fréquents. Dans l'exemple (44), la forme rédupliquée de ḑika "tuer" réfère à la technique de pêche traditionnelle qui consiste à tuer en les frappant des poissons préalablement empoisonnés avec le jus d'une liane toxique. Chaque poisson est tué séparément, avec souvent un certain laps de temps entre deux prises.
(44)$$
\text { mun-a-kom o-dika-dbika-n. }
$$$$
\text { gens-a-PL 3-REDUP-tuer-PL }
$$

$$
\text { Les gens les tuaient et les tuaient. }
$$

Dans la plupart des exemples, le patient semble plutôt devoir être interprété de manière collective comme en (36) et dans les exemples suivants.

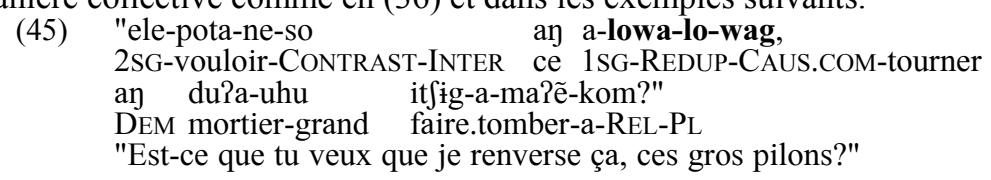




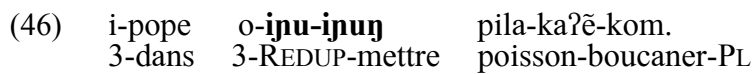

Ils mirent les poissons cuits dedans.

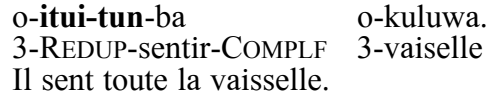

En revanche, certains exemples de réduplication dissyllabique montrent une distribution du sujet, avec une répétition de l'action qui ressemble plus à une répétition interne à l'événement. Nous ne savons pas si cette catégorie de contreexemples doit être mise sur le compte de la neutralisation de la distinction entre les deux types de réduplication traitée plus bas (Cf. 3.4), sur le compte de la distributivité, ou sur celui de l'aspect lexical que nous traitons dans la section suivante.
(48) nani kudgabulu-am o-tui-tuin iki?i. ainsi sirène-TRANSL 3-REDUP-devenir-PL alors C'est ainsi qu'elles sont devenues des sirènes.

(49) beb-a-kom, kofon, ba?e, taitetu, boeuf-a-PL cochon chose pécari.à.collier idka mun-a-kom owi-o-wil-e?e o-ho upi. varié personne-a-PL REDUP-3-monter-INTENS 3-aller le.long.de Les boeufs, les cochons, les trucs, les pakiras, des gens différents montent le long (de la corde).

\subsection{La réduplication comme déclencheur de changement d'aspect lexical}

Au delà du fait que la répétition peut être étudiée sous l'angle de l'aspect (il s'agit bien de la répartition d'un événement dans le temps), l'utilisation d'un processus de pluralisation verbale a souvent comme corrélat un changement dans l'aspect lexical du verbe observé. Il nous semble en effet que le sémantisme particulier d'une occurrence de réduplication en émérillon dépend non seulement du modèle de réduplication utilisé, mais aussi en partie du type de procès dénoté par le verbe et de la quantité des participants (singulier/pluriel, collectif...).

Nous avons vu qu'une conséquence de la répétition interne à l'événement exprimée par la réduplication monosyllabique est de donner un aspect duratif au procès résultant. Une analyse à la Vendler $(1967)^{21}$ montre ainsi que la réduplication monosyllabique a souvent comme effet de "déponctualiser" les procès. Les verbes dénotant habituellement des "achèvements" (procès ponctuels) dénotent donc des "accomplissements" ou des "activités" une fois que la réduplication a rendu le procès non-ponctuel.

${ }^{21}$ Désormais, les termes s'insérant dans une analyse à la Vendler seront introduits entre guillemets : "activité", "accomplissement", "achèvement". 
(50) ãduça wila o-su-su?u

rat bois 3-REDUP-mordre

Le rat a rongé le bois.

(51) mãti-n o-pi-pinõ-ne-o?

INTER-CONT 3-REDUP-péter-CONTRAST-CONT

Pourquoi ne fait-il que péter? (à un moment précis de l'histoire)

La réduplication bisyllabique qui exprime la répétition externe à l'événement, a souvent comme corollaire de rendre le procès atélique (Cf. 2.2). Ainsi, les verbes dénotant habituellement un "accomplissement" ou un "achèvement" expriment souvent, sous cette forme rédupliquée, une "activité" qui se répète un certain temps sans modification.

(52) beçu-enam wãĩwĩ o-enu-ejuy-o22. cassave-CHANG.TOP femme 3-REDUP-mettre-CONT La femme est en train de faire des cassaves.

(53) minawe-zepe, o?u-o-?u wasei. jadis-CONC REDUP-3-manger wasai Pourtant jadis, ils mangeaient souvent du wasai

Enfin, dans la partie suivante, nous verrons que la dichotomie sémantique historique entre les deux types de réduplication des langues tupi-guarani se brouille en synchronie en émérillon.

\subsection{Neutralisation de sens entre réduplication monosyllabique et réduplication dissyllabique}

Nos données montrent par ailleurs que la distinction bien tranchée entre le sémantisme de la réduplication monosyllabique et celui de la réduplication dissyllabique habituellement posée pour les langues tupi-guarani se trouve en réalité brouillée en émérillon.

Dans les faits, si la réduplication monosyllabique est clairement dévolue à l'expression d'une pluralité interne à l'événement, la réduplication dissyllabique qui exprime la plupart du temps une répétition externe à l'événement exprime cependant dans un certain nombre d'exemples une pluralité interne à l'événement. Dans l'exemple suivant, le premier verbe a subi une réduplication

22 De nombreux exemples de réduplication dissyllabique montrent une compatibilité avec l'aspect continu (sous la forme des affixes de constituant $-O$ ou $-n$ ), comme (52), (58) et les deux exemples ci-dessous.

(1) oke-o-kel-o.

REDUP-3-dormir-CONT

Il dort.

(2) o-zau-zaug-o.

3-REDUP-se.baigner-CONT

Ils sont en train de se baigner. 
dissyllabique qui semble devoir être analysée comme le vecteur d'une répétition interne à l'événement. La répétition de l'action «couper quelque chose» à l'intérieur d'un seul intervalle temps crée le sens de «couper quelque chose en morceaux ». Le sens de « couper en morceaux » renvoie donc à un événement unifié, ce que souligne le parallélisme avec les événements " frire », « faire griller », et « manger». L'unité de l'action est renforcée par la particule -?e qui dénote qu'une action similaire a eu lieu précédemment, et c'est seulement cette particule qui véhicule l'idée d'itération de l'événement « couper en morceaux ».
o-eta-eta-?e,
o-bo-siu
o-bo-kach,
o-?u-?e.
3-REDUP-couper-INTENS 3-CAUS-frire 3-CAUS-griller 3-manger-INTENS Il en coupe de nouveau en morceaux, le frit, et le mange une nouvelle fois (le foie).

Voici d'autres exemples de réduplication dissyllabique véhiculant un sens de réduplication interne à l'événement :
(55) tapi?il oPa-o-?al o-ho wila asĩ-natu-mẫe-1-ehe...
tapir REDUP-3-tomber 3-aller bois pointu-bien-REL-RELN-sur Le tapir roula sur les pieux (et mourut percé de toutes parts).
(56) o-pelo-pelog e-iba owa.
3-REDUP-lécher 3-animal 3.visage
Le chien lui lèche le visage.

Aussi peut-on remarquer que certains verbes comme eta «couper», ?u « manger», Pal « tomber » ne sont pas compatibles avec le modèle formel de réduplication monosyllabique. Une fois soumis à la réduplication dissyllabique, ces verbes peuvent rendre soit la réduplication interne à l'événement, soit la réduplication externe à l'événement, comme dans les deux exemples suivants autour du verbe $e t a$ « couper».
$(57)$
$\begin{array}{ll}\text { wã̃ini-enam } & \text { wila-we } \\ \text { femme-CHANG.ToP } & \text { bois-aussi } \\ \text { Quant à la femme, elle coupe du bois. }\end{array}$
o-eta-eta.
3-REDUP-couper

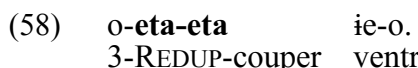
3-REDUP-couper ventre-CONT
Il lui coupe le ventre plusieurs fois (à chaque fois qu'il se fait avaler par l'ogre).

Ces exemples viennent de ce fait contredire une distinction bien tranchée entre les sens des deux modèles de réduplication, distinction qui est affirmée pour certaines langues tupi-guarani et dans la littérature générale sur la famille (Jensen 1990 et 1998 sur la famille tupi-guarani, Seki 2000 sur le kamaiura, Rodrigues 1953 sur le tupinamba et Jensen 1989 sur le wayampi). Cette affirmation ne rend pas compte du fait que la réduplication dissyllabique peut véhiculer une valeur semblable à celle de la réduplication monosyllabique, comme en émérillon ou en wayampi (Jensen 1989, p. 119-121). 
Cette situation peut en fait être expliquée diachroniquement par un abandon de la réduplication monosyllabique comme phénomène productif dans un certain nombre de langues de la famille (Jensen 1990 : 148-149). Notre hypothèse est que dans ces langues-là, la réduplication dissyllabique aurait repris le sémantisme de pluralité interne à l'événement. Ainsi, en émérillon, les occurrences de réduplication monosyllabique sont clairement moins nombreuses que celles de réduplication dissyllabique. De plus, le sémantisme des réduplications monosyllabiques est bien plus homogène que celui des réduplications dissyllabiques. Nous supposons par conséquent que le processus de réduplication monosyllabique est en train de perdre du terrain et celui de réduplication dissyllabique récupère le sémantisme de répétition interne comme une deuxième possibilité, en parallèle au sens de répétition externe. On assiste ainsi à une tendance vers la neutralisation de la distinction sémantique entre répétition interne à l'événement et répétition externe à l'événement, neutralisation déjà effective avec certains verbes.

\section{CONCLUSION}

Cet article décrit le phénomène de réduplication en émérillon en s'attachant à préciser le plus précisément possible les distinctions sémantiques des deux modèles de réduplication de la langue. Cette description s'appuie sur des travaux réalisés à la fois dans les domaines de l'aspect et de la pluralité verbale.

Dans ce cadre-là, il est montré qu'à chaque modèle phonologique de la réduplication est attaché un sens propre : la réduplication monosyllabique traduit une pluralité interne à l'événement alors que la réduplication dissyllabique traduit une pluralité externe à l'événement. Cette affirmation reste à nuancer du fait que ces deux modèles sont selon toute apparence en train de subir une neutralisation. La réduplication monosyllabique perd de sa vitalité et la réduplication dissyllabique reprend en charge le sémantisme de pluralité interne à l'événement en plus de celui de pluralité externe à l'événement.

Outre illustrer le fait que la pluralité verbale peut être exprimée par d'autres moyens que les alternances lexicales ou des marques affixales, en l'occurrence par de la réduplication, cet article pointe un cas où différents modèles de réduplication véhiculent différents sémantismes de répétition. L'un, la répétition interne à l'événement, concerne le déroulement temporel d'un événement conçu comme unique et semble ainsi participer à la notion d'aspect. L'autre, la répétition externe à l'événement, réfère à une multiplicité d'événements discrets. Nous la situons donc volontiers dans le champ de la pluralité verbale. La conceptualisation d'une situation répétitive/répétée en un ou plusieurs événements nous permet de proposer la catégorisation de ce type de répétition comme catégorie aspectuelle ou catégorie de pluralité verbale.

Par ailleurs, il est intéressant d'observer comment s'organise la correspondance entre les deux modèles phonologiques et les deux sémantismes qu'ils induisent. Nous rejoignons ici Rousseau (2002) qui souligne qu'au-delà de l'évidente iconicité entre la forme de la réduplication et son sens répétitif, les distinctions entre les différents types de redoublement peuvent également être 
vues comme iconiques. L'émérillon illustre parfaitement cela : sa réduplication dissyllabique (copiant quasiment toujours l'intégrité de la racine verbale en émérillon) exprime le fait qu'un événement est répété en plusieurs occurrences discrètes, alors que la réduplication monosyllabique (qui ne répète qu'une partie du verbe) exprime de la répétition à l'intérieur d'un seul et même événement. 